\title{
Utilization of DNA-metal Ion Biomatrix as a Relative Humidity Sensor
}

\author{
By Masanori YAMADA* and Taro SUGIYAMA
}

Recently, we prepared the novel biomatrix by the mixing of DNA and metal ion. These biomatrices have a metal ion property, such as oxidative and reducible effects, catalytic action, or the change of coordination number. So, we proposed the humidity sensor for the novel utilization of DNA-metal ion biomatrix. The biomatrix with the humidity property was prepared by the mixing of DNA and metal ion, such as cobalt (II) or nickel (II) ions. These matrices indicated the different color with the change of relative humidity $(\mathrm{RH})$. Especially, the color of $\mathrm{Co}^{2+}$-containing biomatrix changed from blue to red with the increase of RH. These biomatrices may have a potential for the novel RH sensor with flexibility, low cost, nonhazardous, and environmentally benign.

KEY WORDS: DNA / Biopolymers / Matrix / Humidity Sensor / Composites /

DNA, one of the most important materials for the genetic process, can easily be purified from either salmon milts or shellfish gonads, which are generally discarded as industrial waste around the world. In addition, double-stranded DNA has highly specific functions, ${ }^{1,2}$ such as the accumulation of intercalating compounds, the complementary interaction between nucleic acid bases, and the interaction with metal ions. Therefore, the double stranded DNA has been attracted for novel functional materials, such as adsorbents of harmful compounds, ${ }^{3,4}$ electro- and ion-conductive materials, ${ }^{5,6}$ and functional materials. ${ }^{7-9}$ We could also convert the discarded DNA into a novel bio-matrix by the hybridization of DNA and metal ions. ${ }^{10}$ These DNA-metal ion matrices could be created in various desirable forms, such as a gel, capsule, film, or fiber. ${ }^{10}$ Additionally, DNA-metal ion biomatrix indicated the oxidative property. ${ }^{10}$ Metal ions have many functions, such as reductive effect, catalytic action, or the change of coordination number. Therefore, DNA-metal ion biomatrices might be used for various functional materials.

Cobalt (II) and nickel (II) ions can indicate the different color by the change of coordination number with water molecules ${ }^{11,12}$ and this property has been used for the silica gel with the moisture indicator. However, this material is rigid and non-flexible, as a result, the utilized-fields for the moisture indicator or humidity sensor have been limited. So, we propose the relative humidity $(\mathrm{RH})$ sensor for the novel utilization of DNA-metal ion biomatrix that is flexible, non-hazardous, biodegradable, and environmentally benign. These RH sensors with the DNA are novel engineering material and have not been reported as far as we know.

In this paper, we prepared DNA-metal ion biomatrix by the mixing of double-stranded DNA and metal ion, such as cobalt (II) and nickel (II) ions. This matrix had been constructed by the electrostatic interaction between the phosphate group of DNA and metal ion. This DNA-metal ion biomatrix indicated the color change with the RH. Additionally, these color changes is quickly and reversible. Furthermore, the similar phenomena were also obtained at the biomatrix containing a chitosan, one of the famous discarded biopolymers. These biomatrices may have a potential for the novel RH sensor with flexibility, low cost, non-hazardous, and environmentally benign.

\section{EXPERIMENTAL PROCEDURE}

\section{Materials}

Double-stranded DNA (sodium salt from salmon milt, molecular weight; $>5 \times 10^{6}$ ) and chitosan (deacetylated; $80 \%$, molecular weight; $1 \times 10^{5}$ ) was obtained from Yuki Fine Chemical Co., Ltd., Tokyo, Japan and Wako Pure Chemical Industries, Ltd., Osaka, Japan, respectively. Cobalt (II) chloride hexahydrate and nickel (II) chloride hexahydrate was purchased from Kanto Chemical Co., Inc., Tokyo, Japan and Wako Pure Chemical Industries, respectively. All the materials were used without further purification.

\section{Preparation of DNA-metal Ion Biomatrix}

DNA-metal ion composite films were prepared as follows; aqueous DNA solution $(10 \mathrm{mg} / \mathrm{mL})$ and aqueous metal ion solution were mixing in test tube. The mixed solution was applied onto quartz plate $\left(\mathrm{w} 9.9 \times \mathrm{h} 40 \times \mathrm{t} 1 \mathrm{~mm}^{3}\right)$ and dried at room temperature overnight. The molar ratio $(R)$ of DNA and metal ion was determined by the equation (1).

$$
R=\frac{[\text { metal ion }]}{\text { [nucleotide of DNA }]}
$$

where $[$ metal ion $]=\mathrm{a}$ concentration of metal ion and [nucleotide of DNA] = a concentration of nucleotide in DNA-metal ion biomatrix. The $R$ values were $0.01,0.05,0.1$, $0.5,1,5$, and 10 . 


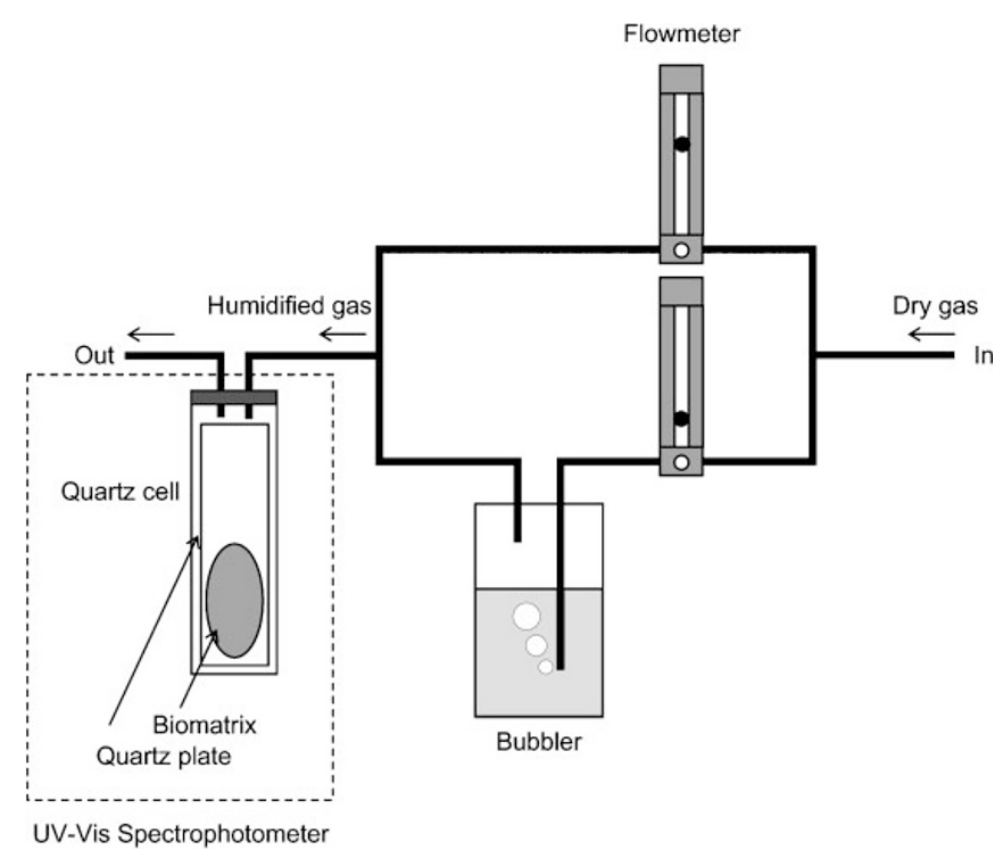

Figure 1. Schematic diagram of the humidity controller with the bubbler. The color change of biomatrix under various RH conditions was detected by the UV-Vis spectrophotometer. The sensor property was demonstrated at room temperature.

\section{Preparation of Chitosan-metal Ion Biomatrix}

Chitosan was dissolved in $1 \% \mathrm{HCl}$ solution. Chitosan-metal ion composite films were prepared as follows; aqueous chitosan solution $(10 \mathrm{mg} / \mathrm{mL})$ and aqueous metal ion solution were mixing in test tube. The mixed solution was applied onto quartz plate and dried at room temperature overnight. The molar ratio $(R)$ of chitosan and metal ion was determined by the equation (2).

$$
R=\frac{[\text { metal ion }]}{\text { [amino group of chitosan] }}
$$

where $[$ metal ion] $=\mathrm{a}$ concentration of metal ion and [nucleotide of DNA] $=$ a concentration of amino group in chitosan-metal ion biomatrix. The $R$ values were $0.01,0.05$, $0.1,0.5,1,5$, and 10 .

\section{Humidity Property of Biopolymer-Metal Ion Biomatrix}

This biomatrix-coated quartz plate set into quartz cell $\left(\mathrm{w} 10 \times \mathrm{h} 40 \times \mathrm{t} 10 \mathrm{~mm}^{3}\right)$. This thickness of matrix was $c a$. $200 \mu \mathrm{m}$. The control of RH was used the equipment with the bubbler (Figure 1). Dried air gas was divided into two sections. One gas flowed through the water phase with the bubbling and made it the saturated vapor (100\% RH). Various humidified gases were prepared by the mixing ratio of $0 \% \mathrm{RH}$ and $100 \% \mathrm{RH}$ gases. The flowing rate was $100 \mathrm{~mL} / \mathrm{min}$. These humidified gases continuously flowed into the quartz cell. The color change of biomatrix was detected by the absorption spectrum using a UV-Vis spectrophotometer U-2010 (Hitachi Co. Ltd., Tokyo, Japan). These measurements of absorption spectra were performed at room temperature.

\section{Characterization of Biopolymer-metal Ion Biomatrix}

The infrared (IR) absorption spectra of the biomatrix were measured by an attenuated total reflection (ATR) methods using a Fourier transform infrared spectrometer FT-IR 8200 (Shimadzu Corp., Kyoto, Japan). These measurements of IR spectra were performed at room temperature. IR samples were prepared as follows; the biopolymer-metal ion mixed solution was applied onto Teflon ${ }^{\circledR}$ plate and dried at room temperature overnight. The biomatrices on Teflon ${ }^{\circledR}$ plate were incubated at the wetting box of $100 \% \mathrm{RH}$ condition for $24 \mathrm{~h}$ and stripped from the plate. The biomatrix at $0 \% \mathrm{RH}$ condition was prepared by the incubation in desiccator with silica gel for $24 \mathrm{~h}$. These spectra were normalization at the $968 \mathrm{~cm}^{-1}$, related to the deoxyribose $\mathrm{C}-\mathrm{C}$ stretching vibration, as the internal reference. ${ }^{13}$ The IR spectrum was measured with the resolution of $4 \mathrm{~cm}^{-1}$.

\section{RESULTS AND DISCUSSION}

The aqueous solution with the mixing of DNA and cobalt (II) chloride was applied onto quarts plate and dried at room temperature overnight. This biomatrix-coated quartz plate set into quartz cell. The control of RH was used the equipment with the bubbler and the humidity gas continuously flowed into the quartz cell. When the humidity gas flowed into the cell, the absorbance of DNA-metal ion biomatrix gradually changed and reached a constant value at a few minutes after the flowing. Therefore, the absorption spectra were measured after the flowing for $10 \mathrm{~min}$ at each $\mathrm{RH}$ condition. Figure 2(a) showed the absorption spectra of DNA-Co ${ }^{2+}(R=0.5)$ biomatrix under various $\mathrm{RH}$ conditions. The absorption spectrum of DNA-Co ${ }^{2+}$ 

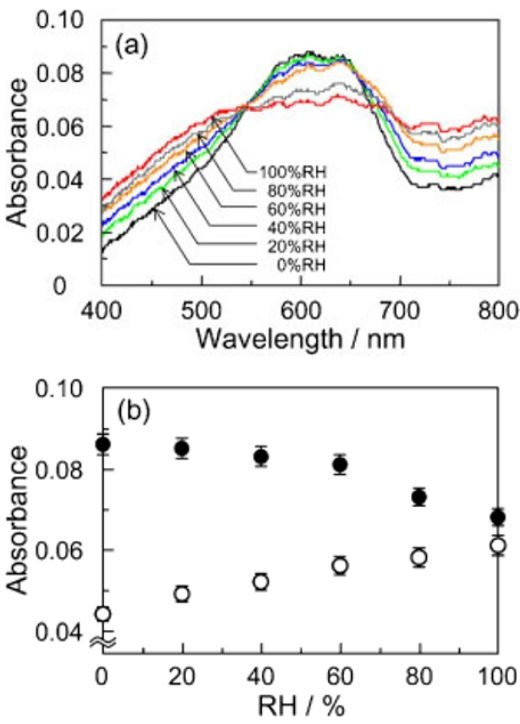

Figure 2. (a) Absorption spectra of DNA-Co ${ }^{2+}(R=0.5)$ biomatrix under various $\mathrm{RH}$ conditions. The $\mathrm{RH}$ range is from $0 \%$ to $100 \%$. (b) Absorbance at $620 \mathrm{~nm}(-)$ and $500 \mathrm{~nm}(O)$ of DNA-Co ${ }^{2+}$ biomatrix under various $\mathrm{RH}$ conditions. The error bar represents the error of $\pm 5 \%$. These measurements of absorption spectra were performed at room temperature.

biomatrix under $0 \% \mathrm{RH}$ condition appeared the maximum absorption peak at $620 \mathrm{~nm}$. The absorption peak at $620 \mathrm{~nm}$ decreased with the increase of $\mathrm{RH}$ and disappeared under $100 \%$ RH condition. Additionally, under high humidity condition, the shoulder peak at $500 \mathrm{~nm}$ appeared. As a result, the color of DNA-Co ${ }^{2+}$ biomatrix changed from blue to red with the increase of RH and these phenomena could also confirm by the viewing. Figure 2(b) showed the change of absorbance at $620 \mathrm{~nm}$ and $500 \mathrm{~nm}$ under various RH values. Since, under humidification, the thickness of biomatrix changed slightly by the transformation of DNA structure, the increase of absorbance at $620 \mathrm{~nm}$ was not linear (please see in Figure 2(b)). The absorbance at $500 \mathrm{~nm}$ linearly increased with the increase of $\mathrm{RH}$ (please see $\bigcirc$ in Figure 2(b)). Therefore, the RH values can estimate from the slop of $500 \mathrm{~nm}$. On the other hand, at the decreasing process of $\mathrm{RH}$ (from $100 \% \mathrm{RH}$ to $0 \% \mathrm{RH}$ ), the changes of absorption spectra were reversible and the error of absorbance with the duplication was $\leq \pm 5 \%$. These results indicate that the color change of $\mathrm{Co}^{2+}$-containing biomatrix by the humidity control is reversible reaction. Additionally, the similar phenomenon was obtained at the chitosan- $\mathrm{Co}^{2+}(R=0.5)$ biomatrix (data not shown).

We prepared the DNA-Ni ${ }^{2+}$ and chitosan- $\mathrm{Ni}^{2+}$ biomatrix by the mixing of aqueous biopolymer and nickel (II) chloride solutions. The color of these metal ion-containing biomatrices changed from yellow to yellowish green with the increase of RH. Figure $3(\bigcirc)$ and $(\bigcirc)$ showed the maximum absorption peak of DNA-Ni ${ }^{2+}(R=0.5)$ and chitosan- $\mathrm{Ni}^{2+}(R=0.5)$ biomatrices under various $\mathrm{RH}$ conditions, respectively. Under the low $\mathrm{RH}$ condition $(\leq 20 \%)$, the maximum absorption peak appeared at approximately $415 \mathrm{~nm}$. This maximum peak was

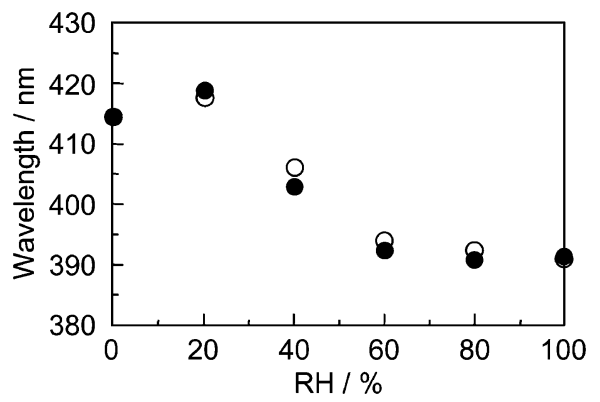

Figure 3. The shift in maximum absorption peak of biopolymer- $\mathrm{Ni}^{2+}$ biomatrix under various $\mathrm{RH}$ conditions. The $\mathrm{RH}$ range is from $0 \%$ to $100 \%$. DNA- $\mathrm{Ni}^{2+}(R=0.5)$ biomatrix; $\bigcirc$, chitosan- $-\mathrm{Ni}^{2+}$ $(R=0.5)$ biomatrix. These measurements of absorption spectra were performed at room temperature.

shifted to lower wavelength with the increase of $\mathrm{RH}$ and reached at the $390 \mathrm{~nm}$ under $100 \% \mathrm{RH}$ condition. Especially, at the $\mathrm{RH}$ ranges of $20 \%-60 \%$, the change of wavelength was ca. $30 \mathrm{~nm}$. Additionally, the error of wavelength with the duplication was similar to the instrumental error of UV-Vis spectrophotometer. In contrast, at the decreasing process of humidity (from $100 \% \mathrm{RH}$ to $0 \% \mathrm{RH}$ ), the changes of absorption peaks were reversible. These results indicate that the color change of $\mathrm{Ni}^{2+}$-containing biomatrix by the humidity control is reversible reaction.

These biopolymer-metal ion biomatrices were stable and did not peel off in our experiments. This stability is due to the effect of metal ion, such as $\mathrm{Co}^{2+}$ or $\mathrm{Ni}^{2+}$. Since DNA and quartz are negatively charged, the repulsive force occurs between DNA and quartz. However, in our DNA-metal ion biomatrices, metal ion can connect the DNA and quartz by the electrostatic interaction. Additionally, we demonstrated the test of $\mathrm{RH}$ sensor at the biopolymer-metal ion biomatrix with various $R$ values. At the $R<0.5$, the absorbance of biomatrix was low and could not use for the RH sensor. Additionally, at the $R \geq 5$, these biomatrices showed the crystallization of metal chloride in the part of film and these biomatrices were not flexible. Therefore, the property of RH sensor can estimate at the $R$ values of $0.5-1$. On the other hand, we prepared DNA-metal ion biomatrices with various thicknesses. However, at the thickness of $<200 \mu \mathrm{m}$, the absorbance of DNAmetal ion biomatrix was too low to detect the sensor property. Additionally, at the thickness of $>200 \mu \mathrm{m}$, there was uneven in DNA-metal ion film, as a result, the absorption spectra with the reappearance did not obtain. Therefore, in this experiment, we have used the DNA-metal ion biomatrices of ca. $200 \mu \mathrm{m}$.

The molecular structures of DNA- $\mathrm{Co}^{2+}$ biomatrix were characterized by an infrared (IR) spectroscopic analysis with the attenuated total reflection (ATR) prism. IR samples were prepared by the incubation under the wetting box of $100 \% \mathrm{RH}$ condition for $24 \mathrm{~h}$. These IR samples were taken out from the box and measured within $30 \mathrm{~s}$. So, the IR spectra indicate the molecular structure of DNA-Co ${ }^{2+}$ biomatrix under $100 \% \mathrm{RH}$ condition. Figure 4 showed the IR spectra of $\mathrm{DNA}^{-\mathrm{Co}^{2+}}$ 


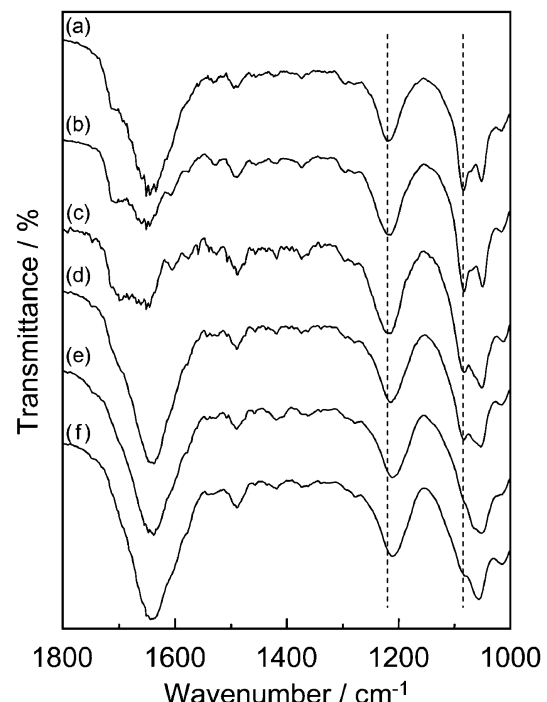

Figure 4. IR spectra of DNA-Co ${ }^{2+}$ biomatrix with various $R$ values under $100 \% \mathrm{RH}$ condition. IR samples were taken out from the wetting box of $100 \% \mathrm{RH}$ and measured within $30 \mathrm{~s}$. (a), DNA; (b), $R=$ 0.01 ; (c), $R=0.05$; (d), $R=0.5$; (e), $R=5$; (f), $R=10$. These measurements of IR spectra were performed at room temperature.

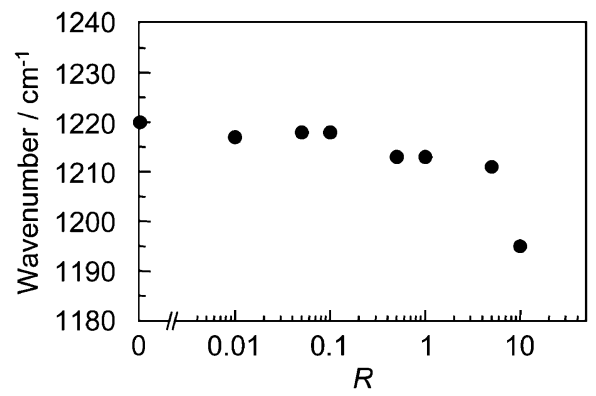

Figure 5. The shift of transmittance peak in the phosphate group of DNA$\mathrm{Co}^{2+}$ biomatrix with various $R$ values. This absorption peak at $1220 \mathrm{~cm}^{-1}$ is related to the antisymmetric vibration of the phosphate group.

biomatrix with various $R$ values. The absorption band at $1220 \mathrm{~cm}^{-1}$, related to the antisymmetric vibration of the phosphate group, ${ }^{10,13-16}$ was shifted to a lower wavenumber with the increase of the $R$ values. Furthermore, the absorption band at $1090 \mathrm{~cm}^{-1}$, the symmetric stretching vibration of phosphate group, ${ }^{10,13-16}$ relatively decreased with an increase of the $R$ values. Figure 5 showed the wavenumber of absorption band for the phosphate group with various $R$ values. When the $R$ value increased, the absorption band of the phosphate group $\left(1220 \mathrm{~cm}^{-1}\right)$ shifted to a lower wavenumber and reached at $1195 \mathrm{~cm}^{-1}$. Similar phenomenon has been reported at the interaction of heavy metal ion and the UV-irradiated DNA film. ${ }^{17}$ These results suggest that $\mathrm{Co}^{2+}$ strongly bind to the phosphate group by an electrostatic interaction. Similar molecular structures were also suggested at the DNA-Ni ${ }^{2+}$ biomatrix (data not shown). In contrast, since the absorption band at $1600-1700 \mathrm{~cm}^{-1}$, related to the stretching vibration of $\mathrm{C}=\mathrm{O}$ and $\mathrm{C}=\mathrm{C}$ of nucleic acid

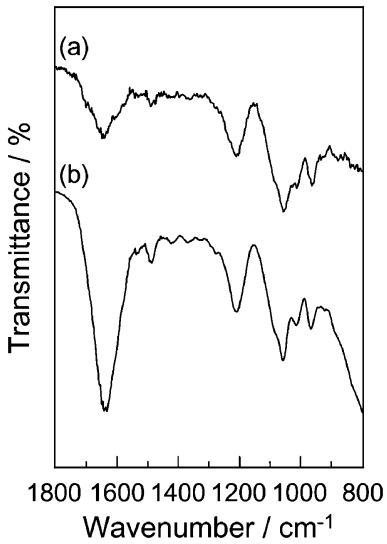

Figure 6. IR spectra of DNA-Co ${ }^{2+}(R=0.5)$ biomatrix under (a) $0 \% \mathrm{RH}$ and (b) $100 \% \mathrm{RH}$ conditions. These measurements of IR spectra were performed at room temperature.

bases ${ }^{16}$ increased with the increase of $R$ values. In addition, the pure cobalt chloride under $100 \% \mathrm{RH}$ condition also showed the strong absorption band at $1650 \mathrm{~cm}^{-1}$. Therefore, the increase of intensity at $1650 \mathrm{~cm}^{-1}$ with the increase of $R$ value is not due to the interaction with the nucleic acid bases, but due to the hydrated-cobalt (II) chloride.

Figure 6(a) and 6(b) showed the IR spectra of DNA-Co ${ }^{2+}$ biomatrix $(R=0.5)$ under $0 \% \mathrm{RH}$ and $100 \% \mathrm{RH}$ conditions, respectively. The absorption band at $1250-1500 \mathrm{~cm}^{-1}$, which can be attributed to the nucleic acid base ${ }^{18}$ relatively increased with the humidity. This is due to the interaction of nucleic acid base with the hydrated-cobalt ion. The increase of intensity based on the interaction with DNA and metal ion has been reported at the IR spectra in aqueous solution. ${ }^{13,15,18}$ Additionally, the conformational change of DNA structure with the $\mathrm{RH}$ has been reported. ${ }^{19-21}$ In this case, DNA structure changes from A-form to B-form with the increase of RH. Therefore, the coordinated structures around the metal ion change under the differential RH conditions. These results suggest that the conformational change of DNA structure occurs at the high humidity condition and the $\mathrm{Co}^{2+}$ in $\mathrm{DNA}-\mathrm{Co}^{2+}$ biomatrix have a strong interactions with not only the phosphate groups but also the nucleic acid bases. Furthermore, the absorption band of $1050 \mathrm{~cm}^{-1}, \mathrm{Co}^{2+}-\mathrm{OH}$ bending mode, ${ }^{22}$ relatively increased in comparison with the stretching vibration of deoxyribose at $968 \mathrm{~cm}^{-1}$. Therefore, under $100 \% \mathrm{RH}$ condition, $\mathrm{Co}^{2+}$ in biomatrix has also interacted with water molecules.

We also demonstrated the IR measurements of chitosanmetal ion biomatrix under $100 \%$ RH condition. As a result, the absorption band based on amino group of chitosan shifted to a lower wavenumber with the increase of $R$ values (data not shown). Therefore, chitosan-metal ion biomatrices have been formed by the interaction between the amino group of chitosan and metal ion.

Generally, the color change based on the RH is due to the difference of the coordination number around the metal ion. 
The coordinated structure of $\mathrm{Co}^{2+}$ under non-humidity, such as $0 \% \mathrm{RH}$ condition, is square-planar. ${ }^{11,23}$ In contrast, the structure of $\mathrm{Co}^{2+}$ under high humidity condition, such as $100 \%$ $\mathrm{RH}$, has constructed the octahedral with two water molecules. ${ }^{11,23}$ As a result, the color of $\mathrm{Co}^{2+}$-containing material, such as silica gel with the moisture indicator, can change from blue to red with the increase of the RH value. The similar coordination structure is likely to be constructed in DNA-Co ${ }^{2+}$ biomatrices. In this case, the $\mathrm{Co}^{2+}$ is surrounded with some phosphate groups or nucleic acid bases in DNA biomatrix (see the IR spectra in Figures 4 and 6). As a result, the DNA-Co ${ }^{2+}$ biomatrix indicated the color change from blue to red with the increase of RH. Since this color change was based on the characteristic of metal ion, DNA-Ni ${ }^{2+}$ biomatrix showed the color change different from DNA-Co ${ }^{2+}$ biomatrix. In contrast, the different color of chitosan-metal ion biomatrix with the change of RH values can also explain at the similar phenomena.

In conclusion, the biomatrix with the humidity property was prepared by the mixing of DNA and metal ion, such as $\mathrm{Co}^{2+}$ or $\mathrm{Ni}^{2+}$. These biomatrices was constructed by the electrostatic interaction to the phosphate group and the coordination interaction to the nucleic acid base. Additionally, these biomatrices indicated the different color by the coordination number of water molecule around the metal ion in biomatrix. Especially, the color of $\mathrm{Co}^{2+}$-containing biomatrix changed from blue to red with the increase of RH. These biomatrices may have a potential for the novel $\mathrm{RH}$ sensor with flexibility, low cost, non-hazardous, and environmentally benign.

Acknowledgements. This work was supported by the Grants-in-Aid for Scientific Research from the Ministry of Education, Culture, Sports, Science and Technology of Japan (No. 19750187).

\section{REFERENCES}

1. W. Saenger, "Principles of Nucleic Acid Structure," Springer-Verlag, Berlin, 1987.

2. M. J. Waring, Annu. Rev. Biochem., 50, 159 (1981).

3. M. Yamada, K. Kato, M. Nomizu, K. Ohkawa, H. Yamamoto, and N. Nishi, Environ. Sci. Technol., 36, 949 (2002).

4. K. Iwata, T. Sawadaishi, S. Nishimura, S. Tokura, and N. Nishi, Int. J. Biol. Macromol., 18, 149 (1996).

5. H. Nakayama, H. Ohno, and Y. Okahata, Chem. Commum., 2300 (2001).

6. N. Nishimura and H. Ohno, J. Chem. Mater., 12, 2299 (2002).

7. B. Zhao, B. Wang, Y. Zhao, S. Zhang, N. Sakairi, and N. Nishi, Int. J. Biol. Macromol., 37, 143 (2005).

8. K. Yang, B. Zheng, F. Li, X. Wen, and C. Zhao, Desalination, 175, 297 (2005).

9. B. Fugetsu, S. Satoh, T. Shiba, T. Mizutani, Y. Nodasaka, K. Yamazaki, K. Shimizu, M. Shindoh, K. Shibata, N. Nishi, Y. Sato, K. Tohji, and F. Watari, Bull. Chem. Soc. Jpn., 77, 1945 (2004).

10. M. Yamada, M. Yokota, M. Kaya, S. Satoh, B. Jonganurakkun, M. Nomizu, and N. Nishi, Polymer, 46, 10102 (2005).

11. L. I. Katzin and J. R. Ferraro, J. Am. Chem. Soc., 74, 2752 (1952).

12. H. Yajima, T. Miyamoto, R. Endo, and S. Furuya, Kobunshi Ronbunshu, 46, 577 (1989).

13. H. Arakawa, R. Ahmad, M. Naoui, and H. A. Tajmir-Riahi, J. Biol. Chem., 275, 10150 (2000).

14. E. V. Hackl, S. V. Kornilova, and Y. P. Blagoi, Int. J. Biol. Macromol., 35, 175 (2005).

15. H. A. Tajmir-Riahi, M. Naoui, and R. Ahimad, Biopolymers, 33, 1819 (1993).

16. M. Banyay, M. Sarkar, and A. Gräslund, Biophys. Chem., 104, 477 (2003).

17. M. Yamada, K. Kato, M. Nomizu, M. Haruki, K. Ohkawa, H. Yamamoto, and N. Nishi, Bull. Chem. Soc. Jpn., 75, 1627 (2002).

18. E. V. Hackl, S. V. Kornilova, L. E. Kapinos, V. V. Andrushchenko, V. L. Galkin, D. N. Grigoriev, and Y. P. Blagoi, J. Mol. Struct., 408409, 229 (1997).

19. M. J. B. Tunis-Schneider and M. F. Maestre, J. Mol. Biol., 52, 521 (1970).

20. M. F. Maestre, J. Mol. Biol., 52, 543 (1970).

21. V. N. Potaman, D. G. Alexeev, I. Y. Skuratovskii, A. Z. Rabinovich, and L. S. Shlyakhtenko, Nucleic Acids Res., 9, 55 (1981).

22. K. Nakamoto, "Infrared Spectra of Inorganic and Coordination Compounds," J. Wiley \& Son, New York, 1968.

23. F. A. Cotton and G. Wilkinson, "Advanced inorganic chemistry," 4th ed., J. Wiley \& Son, New York, 1980. 